\title{
Complex conductivity in strongly fluctuating layered superconductors
}

\author{
B.D. Tinh, L.M. Thu, L.V. Hoa \\ Department of Physics, Hanoi National University of Education, 136 Xuanthuy, Caugiay, Hanoi, Vietnam
}

Received October 20, 2013, in final form February 7, 2014

\begin{abstract}
The time-dependent Ginzburg-Landau approach is used to calculate the complex fluctuation conductivity in layered type-II superconductor under magnetic field. Layered structure of the superconductor is accounted for by means of the Lawrence-Doniach model, while the nonlinear interaction term in dynamics is treated within self-consistent Gaussian approximation. In high- $T_{\mathrm{C}}$ materials, large portion of the $H-T$ diagram belongs to vortex liquid phase. The expressions summing contributions of all the Landau levels are presented in explicit form which are applicable essentially to the whole phase and are compared to experimental data on high- $T_{\mathrm{C}}$ superconductor $\mathrm{YBa}_{2} \mathrm{Cu}_{3} \mathrm{O}_{7-\delta}$. Above the crossover to the "normal phase", our results agree with the previously obtained.
\end{abstract}

Key words: time-dependent Ginzburg-Landau, complex conductivity, type-II superconductor

PACS: $74.40 . G h, 74.25 . f c, 74.20 . D e$

\section{Introduction}

There has been a renewed interest in the effect of strong thermal fluctuations in layered high $T_{\mathrm{C}}$ superconductors that exhibit Nernst effect [1] well above $T_{\mathrm{c}}$. The experiments were interpreted microscopically as due to virtual (preformed) pairs [2], although can be described on the "mesoscopic" level using Ginzburg-Landau approach [3]. This indicates large renormalization of $T_{\mathrm{c}}$ (of order 1) by thermal fluctuations in strongly layered materials like $\mathrm{Bi}_{2} \mathrm{Sr}_{2} \mathrm{CaCuO}_{8+\delta}$ and $\mathrm{La}_{2-} \mathrm{Sr}_{x} \mathrm{CuO}_{4}$. Microscopic parameters of the material determine both the actual $T_{\mathrm{c}}$ at which the Cooper pairs are coherent and the mean field critical temperature $T_{\mathrm{c}}^{\mathrm{MF}}$ until which incoherent Cooper pairs exist and effect the transport, magnetism and thermodynamics of the material. More recently strong diamagnetism was also observed [4], although it is still debated on experimental level. All these new results are concerned with DC transport.

The AC transport in strongly fluctuating type-II superconductors have been a subject for active research for many years, both theoretically and experimentally. While the seminal calculation of the enhancement of the DC conductivity in the normal phase due to virtual Cooper pair created by thermal fluctuations by Aslamazov and Larkin was done in the framework of the microscopic BCS theory [5], the approach becomes cumbersome in more complicated situations involving external magnetic field, layerred structure, etc. As usual in these circumstances (especially in the absense of a simple accepted microscopic model for high $T_{\mathrm{c}}$ and other recently discovered "unconventional" strongly fluctuating superconductors), a more phenomenological Ginzburg-Landau approach adapted to incorporate thermal fluctuations turns out to be more effective [5, 6]. A general method to model the thermal fluctuations in dynamics is to add a random Langevin white noise to the time-dependent Ginzburg-Landau (TDGL) equations. The transport coefficients within this approach are obtained as a long time limit of a driven system. The model, therefore, becomes rather complicated and approximations should be made. In the gaussian fluctuations regime (in the normal phase not very close to criticality, where the quartic term in the GL free energy is dominant), the expressions for complex conductivity at zero magnetic field have been obtained very early on [7, 8]. This was expanded later by Dorsey and coworkers [9, 10] to include the critical fluctuations region by a variety of nonperturbative methods (Hartree approximation, large 
number of components $N$ limit, $\varepsilon$-expansion. The results were in line [9] with general physical scaling arguments by Fisher, Fisher, and Huse [11].

The complex conductivity in magnetic field in the normal phase was calculated using TDGL equation by Larkin and Varlamov [5]. The general expression valid for complex conductivity in layered superconductors under the assumption of gaussian fluctuations (neglecting the quartic in the order parameter term in the GL free energy) was also calculated and presented in [5] as a sum over all the Landau levels. On the opposite side of the phase diagram, namely in a strongly pinned case (vortex glass and Bragg glass), the same quantity was calculated using both macroscopic elastic theory [12] and the TGDL [13]. In yet another limit of the Abrikosov lattice phase of a clean superconductor, see [6], the complex conductivity was recently calculated [14] (within the lowest Landau level). The present work is complementary to all these in that we concentrate on the thermally depinned homogeneous phase marked as "vortex liquid" [6]. The complex conductivity in magnetic field in 2D and 3D was calculated using TDGL equation [15, 16]. In strongly layered high $T_{\mathrm{c}}$ materials, this portion of the magnetic phase diagram is very large and consequently well studied experimentally [17-19]. In this region, thermal fluctuations are so strong that one cannot neglect the quartic in the order parameter term of the GL energy. This term, however, can be incorporated self-consistently into the framework of [5].

In this paper, the complex conductivity including all Landau levels is calculated in a layered superconductor under magnetic field in the vortex liquid phase by using TDGL approach with thermal fluctuations modelled by the Langevin white noise. We obtain an expression summing all Landau levels in an explicit form. The rest of the paper is organized as follows. In section 2, the Lawrence-Donich model in its time dependent form is briefly recalled and the main assumptions are specified. In section 3 , the interaction term in dynamics is treated within self-consistent gaussian approximation sufficient for description of the vortex liquis. The complex conductivity calculation within the same approximation is the subject of section 4. The results are compared with experimental data on HTSC in section 5, while the work is summarized in section 6 .

\section{Thermal fluctuations in the time dependent GL Lawrence-Doniach model}

Cooper pairing in layered superconductors can be described by the 2D distribution of the order parameter $\Psi_{n}(\mathbf{r})$ in each of the layers labeled by $n$. The Lawrence-Doniach version of the GL free energy includes the Josephson coupling between the layers [5]:

$$
F_{\mathrm{GL}}=s^{\prime} \sum_{n} \int \mathrm{d}^{2} r\left(\frac{\hbar^{2}}{2 m^{*}}\left|\mathbf{D} \Psi_{n}\right|^{2}+\frac{\hbar^{2}}{2 m_{\mathrm{c}} d^{\prime 2}}\left|\Psi_{n}-\Psi_{n+1}\right|^{2}+a\left|\Psi_{n}\right|^{2}+\frac{b^{\prime}}{2}\left|\Psi_{n}\right|^{4}\right) .
$$

Here, $s^{\prime}$ is the order parameter effective "thickness" and $d^{\prime}>s^{\prime}$ is the distance between layers. The Lawrence-Doniach model approximates the paired electrons density of states by homogeneous infinitely thin planes separated by distance $d^{\prime}$. For simplicity, we assume $a=\alpha T_{\mathrm{c}}^{\mathrm{MF}}(t-1), t^{\mathrm{MF}} \equiv T / T_{\mathrm{c}}^{\mathrm{MF}}$, although this temperature dependence can be easily modified to better describe the experimental coherence length. The "mean field" critical temperature $T_{\mathrm{c}}^{\mathrm{MF}}$ depends on UV cutoff and is often much larger than "renormalized" critical temperature $T_{\mathrm{c}}$. This temperature is significantly higher than the measured critical temperature $T_{\mathrm{c}}$ due to strong thermal fluctuations on the mesoscopic scale.

The covariant derivatives are defined by $\mathbf{D} \equiv \boldsymbol{\nabla}+\mathbf{i}\left(2 \pi / \Phi_{0}\right) \mathbf{A}$, where the vector potential describes a constant and homogeneous magnetic field $\mathbf{A}=(-B y, 0)$ and $\Phi_{0}=h c / e^{*}$ is the flux quantum with $e^{*}=2|e|$. The two scales, the coherence length $\xi^{2}=\hbar^{2} /\left(2 m^{*} \alpha T_{\mathrm{c}}\right)$, and the penetration depth, $\lambda^{2}=$ $c^{2} m^{*} b^{\prime} /\left(4 \pi e^{* 2} \alpha T_{\mathrm{c}}\right)$ define the GL ratio $\kappa \equiv \lambda / \xi$, which is very large for HTSC. In the case of strongly type-II superconductors, the magnetization is by a factor $\kappa^{2}$ smaller than the external field for magnetic field larger than the first critical field $H_{\mathrm{cl}}(T)$, so that we take $B \approx H$. The electric current, $\mathbf{J}=\mathbf{J}^{\mathrm{n}}+\mathbf{J}^{\mathrm{s}}$, includes both the Ohmic normal part

$$
\mathbf{J}^{\mathrm{n}}=\sigma_{n} \mathbf{E},
$$

and the supercurrent

$$
\mathbf{J}_{n}^{\mathrm{s}}(\mathbf{r})=\frac{\mathrm{i} e^{*} \hbar}{2 m^{*}}\left(\Psi_{n}^{*} \mathbf{D} \Psi_{n}-\Psi_{n} \mathbf{D} \Psi_{n}^{*}\right)
$$


Since we are interested in a transport phenomenon, it is necessary to introduce a dynamics of the order parameter. The simplest one is a gauge-invariant version of the "type A" relaxational dynamics [20]. In the presence of thermal fluctuations, which on the mesoscopic scale are represented by a complex white noise, it reads:

$$
\frac{\hbar^{2} \gamma^{\prime}}{2 m^{*}} D_{\tau} \Psi_{n}=-\frac{1}{s^{\prime}} \frac{\delta F_{\mathrm{GL}}}{\delta \Psi_{n}^{*}}+\zeta_{n},
$$

with correlator

$$
\left\langle\zeta_{n}(\mathbf{r}, \tau), \zeta_{m}\left(\mathbf{r}^{\prime}, \tau^{\prime}\right)\right\rangle=\frac{\hbar^{2} \gamma^{\prime} T}{m^{*} s^{\prime}} \delta_{n m} \delta\left(\mathbf{r}-\mathbf{r}^{\prime}\right) \delta\left(\tau-\tau^{\prime}\right) .
$$

Here, $D_{\tau} \equiv \partial / \partial \tau-\mathrm{i}\left(e^{*} / \hbar\right) \Phi$ is the covariant time derivative, with $\Phi=-E_{\tau} y$ being the scalar electric potential describing the driving force in a purely dissipative dynamics. The electric field is, to a good approximation in the vortex liquid phase coordinate, independent (at least for frequences below $\mathrm{THz}$ range, see argumentation in [21]), but is a monorchromatic periodic function of time

$$
E_{x}=0, \quad E_{y}(\tau)=E \exp (-\mathrm{i} \omega \tau) .
$$

Throughout most of the paper we use the coherence length $\xi$ as a unit of length and $H_{\mathrm{c} 2}=\Phi_{0} / 2 \pi \xi^{2}$ as a unit of the magnetic field, with dimensionles field $b=B / H_{\mathrm{c} 2}$. In analogy to the coherence length, one defines a characteristic time scale: the GL "relaxation" time $\tau_{\mathrm{GL}}=\gamma^{\prime} \xi^{2} / 2$. Similarly, it is convenient to use the following unit of the electric field, $E_{\mathrm{GL}}=H_{\mathrm{c} 2} \xi / c \tau_{\mathrm{GL}}$, so that the dimensionless field is $\mathscr{E}=E / E_{\mathrm{GL}}$. The dynamical equation, equation [2.4), written in dimensionless units reads:

$$
\left(D_{\tau}-\frac{1}{2} D^{2}\right) \psi_{n}+\frac{1}{2 d^{2}}\left(2 \psi_{n}-\psi_{n+1}-\psi_{n-1}\right)-\frac{1-t^{\mathrm{MF}}}{2} \psi_{n}+\left|\psi_{n}\right|^{2} \psi_{n}=\bar{\zeta}_{n},
$$

where $d=d^{\prime} / \xi_{z}$ is dimensionless layer distance. The coherence length perpendicular to the layers is smaller compared to $\xi$ by the anisotropy parameter $\gamma[6]$.

The covariant time derivatives become $D_{\tau}=\partial / \partial \tau+\mathrm{i} \mathscr{E}(\tau) y$, the covariant derivatives are defined by $D_{x}=\partial / \partial x-\mathrm{i} b y, D_{y}=\partial / \partial y$. The "mean field" critical temperature $T_{\mathrm{c}}^{\mathrm{MF}}$ depends on the ultraviolet (UV) cutoff. This temperature is higher than the measured critical temperature $T_{\mathrm{c}}$ due to strong thermal fluctuations on the mesoscopic scale, and it will be renormalized later. The dimensionless Langevin white-noise forces, $\bar{\zeta}_{n}=b^{\prime 1 / 2}\left(2 \alpha T_{\mathrm{c}}^{\mathrm{MF}}\right)^{-3 / 2} \zeta_{n}$, are correlated through $\left\langle\bar{\zeta}_{n}^{*}(\mathbf{r}, \tau) \bar{\zeta}_{m}\left(\mathbf{r}^{\prime}, \tau^{\prime}\right)\right\rangle=2 \eta t / s \delta_{n m} \delta\left(\mathbf{r}-\mathbf{r}^{\prime}\right) \delta\left(\tau-\tau^{\prime}\right)$, with a dimensionless fluctuation strength parameter related to the well known Ginzburg number [5, 6] by

$$
\eta=\pi \sqrt{2 \mathrm{Gi}}, \quad \mathrm{Gi}=\frac{1}{2}\left(\frac{8 e^{2} \kappa^{2} \xi T_{\mathrm{c}}^{\mathrm{MF}} \gamma}{c^{2} \hbar^{2}}\right)^{2} .
$$

The dimensionless current density is $\mathbf{J}^{\mathrm{s}}=J_{\mathrm{GL}} \mathbf{j}^{\mathrm{s}}$, where

$$
\mathbf{j}_{n}^{\mathbf{s}}=\frac{\mathbf{i}}{2}\left(\psi_{n}^{*} \mathbf{D} \psi_{n}-\psi_{n} \mathbf{D} \psi_{n}^{*}\right)
$$

with $J_{\mathrm{GL}}=c H_{\mathrm{C} 2} /\left(2 \pi \xi \kappa^{2}\right)$ being the unit of the current density. Consistently, the conductivity will be given in the units of

$$
\sigma_{\mathrm{GL}}=\frac{J_{\mathrm{GL}}}{E_{\mathrm{GL}}}=\frac{c^{2} \gamma^{\prime}}{4 \pi \kappa^{2}}
$$

This unit is close to the normal state conductivity $\sigma_{n}$ in dirty limit superconductors [22]. In general, there is a factor $k$ of the order one relating the two: $\sigma_{n}=k \sigma_{\mathrm{GL}}$.

\section{The Green's function of TDGL in Gaussian approximation}

Let us first assume that the vortex liquid is not driven by the electric field. As mentioned above, the cubic term in the TDGL equation (2.7) can be treated in the self-consistent gaussian approximation (explained in detail in [23]) by replacing $\left|\psi_{n}\right|^{2} \psi_{n}$ with a linear one $2\left\langle\left|\psi_{n}\right|^{2}\right\rangle \psi_{n}$

$$
\left(\frac{\partial}{\partial \tau}-\frac{1}{2} D^{2}-\frac{b}{2}\right) \psi_{n}+\frac{1}{2 d^{2}}\left(2 \psi_{n}-\psi_{n+1}-\psi_{n-1}\right)+\varepsilon \psi_{n}=\bar{\zeta}_{n}
$$


Here, the value of the coefficient of the linear term,

$$
\varepsilon=-\frac{1-t^{\mathrm{MF}}-b}{2}+2\left\langle\left|\psi_{n}\right|^{2}\right\rangle
$$

is different from the noninteracting one.

The relaxational linearized TDGL equation with a Langevin noise, equation [3.1), is solved using the retarded (vanishing for $\tau<\tau^{\prime}$ ) Green function (GF) $G_{k_{z}}^{0}\left(\mathbf{r}, \tau ; \mathbf{r}^{\prime}, \tau^{\prime}\right)$ :

$$
\psi_{n}(\mathbf{r}, \tau)=\int_{0}^{2 \pi / d} \frac{\mathrm{d} k_{z}}{2 \pi} \mathrm{e}^{-\mathrm{i} n k_{z} d} \int \mathrm{d} \mathbf{r}^{\prime} \int \mathrm{d} \tau^{\prime} G_{k_{z}}^{0}\left(\mathbf{r}, \tau ; \mathbf{r}^{\prime}, \tau^{\prime}\right) \bar{\zeta}_{k_{z}}\left(\mathbf{r}^{\prime}, \tau^{\prime}\right) .
$$

The GF satisfies

$$
\left\{\frac{\partial}{\partial \tau}-\frac{1}{2} D^{2}-\frac{b}{2}+\frac{1}{d^{2}}\left[1-\cos \left(k_{z} d\right)\right]+\varepsilon\right\} G_{k_{z}}^{0}\left(\mathbf{r}, \mathbf{r}^{\prime}, \tau-\tau^{\prime}\right)=\delta\left(\mathbf{r}-\mathbf{r}^{\prime}\right) \delta\left(\tau-\tau^{\prime}\right) .
$$

The GF is a Gaussian

$$
G_{k_{z}}^{0}\left(\mathbf{r}, \mathbf{r}^{\prime}, \bar{\tau}\right)=\theta(\bar{\tau}) C_{k_{z}} \exp \left[\frac{\mathrm{i} b}{2} X\left(y+y^{\prime}\right)\right] \exp \left(-\frac{X^{2}+Y^{2}}{2 \beta}\right),
$$

where $X=x-x^{\prime}, Y=y-y^{\prime}, \bar{\tau}=\tau-\tau^{\prime} . \theta(\bar{\tau})$ is the Heaviside step function, $C$ and $\beta$ are coefficients.

Substituting equation (3.5) into equation (3.4), one obtains:

$$
\begin{gathered}
\beta=\frac{2}{b} \tanh (b \bar{\tau} / 2), \\
C=\frac{b}{4 \pi} \exp \left(-\left\{\varepsilon-\frac{b}{2}+\frac{1}{d^{2}}\left[1-\cos \left(k_{z} d\right)\right]\right\} \bar{\tau}\right)\left[\sinh \left(\frac{b \bar{\tau}}{2}\right)\right]^{-1} .
\end{gathered}
$$

The thermal average of the density of Cooper pairs can be expressed via the Green's functions:

$$
\begin{aligned}
\left\langle\left|\psi_{n}(\mathbf{r}, \tau)\right|^{2}\right\rangle & =2 \omega t \frac{d}{s} \int_{0}^{2 \pi / d} \frac{\mathrm{d} k_{z}}{2 \pi} \int \mathrm{d} \mathbf{r}^{\prime} \int \mathrm{d} \tau^{\prime}\left|G_{k_{z}}^{0}\left(\mathbf{r}-\mathbf{r}^{\prime}, \tau-\tau^{\prime}\right)\right|^{2} \\
& =\frac{\omega t b}{2 \pi s} \int_{\bar{\tau}=\tau_{\mathrm{c}}}^{\infty} \frac{f(\varepsilon, \bar{\tau})}{\sinh (b \bar{\tau})}
\end{aligned}
$$

where

$$
f(\varepsilon, \bar{\tau})=\exp \left[\frac{2 v^{2}}{b} \tanh \left(\frac{b \bar{\tau}}{2}\right)\right] \mathrm{e}^{-\left(2 \varepsilon-b+v^{2}\right) \bar{\tau}} \mathrm{e}^{-2 \bar{\tau} / d^{2}} I_{0}\left(2 \bar{\tau} / d^{2}\right) .
$$

Substituting the density of Cooper pairs (3.8) into equation (3.2) and after renormalization [23], the latter takes the form

$$
\varepsilon=-\frac{1-b-t}{2}-\frac{\eta t}{\pi s} \int_{0}^{\infty} \mathrm{d} \bar{\tau} \ln [\sinh (b \bar{\tau})] \frac{\mathrm{d}}{\mathrm{d} \bar{\tau}}\left[\frac{g(\varepsilon, \bar{\tau})}{\cosh (b \bar{\tau})}\right]+\frac{\omega t}{\pi s}\left[\gamma_{\mathrm{E}}-\ln \left(b d^{2}\right)\right],
$$

where $g(\varepsilon, \bar{\tau})=\mathrm{e}^{-\left(2 \varepsilon-b+2 / d^{2}\right) \bar{\tau}} I_{0}\left(2 \bar{\tau} d^{-2}\right)$ with $I_{0}(x)=(1 / 2 \pi) \int_{0}^{2 \pi} \mathrm{e}^{x \cos \theta} \mathrm{d} \theta$ being the modified Bessel function, $\bar{\tau}=\tau-\tau^{\prime}, t=T / T_{\mathrm{c}}$, and $\gamma_{\mathrm{E}}=0.577$ is Euler constant. As was discussed in [23] there are UV divergences in the intermediate steps, equation (3.2), that are regularized by a cutoff. There is a degree of arbitrariness in its choice and it was shown that in the TDGL theory, the cutoff having a dimension of time, $\tau_{c}$, rather than energy, is most convenient. However, in the renormalized equation, equation (3.10), the cutoff does not appear: the mean field temperature $T_{\mathrm{c}}^{\mathrm{MF}}$ is now replaced by $T_{\mathrm{c}}$. Having determined the GF, one can use it to calculate the complex conductivity. 


\section{The complex conductivity of a layered superconductor}

After the thermal averaging of the supercurrent density at time $\tau$, defined by equation (2.9), it can be expressed via the Green's functions as follows:

$$
j_{y}^{\mathrm{s}}(\tau)=\mathrm{i} \eta t \frac{d}{s} \int_{0}^{2 \pi / d} \frac{\mathrm{d} k_{z}}{2 \pi} \int_{r^{\prime}, \tau^{\prime}} G_{k_{z}}^{*}\left(\mathbf{r}, \mathbf{r}^{\prime}, \tau-\tau^{\prime}\right) \frac{\partial}{\partial y} G_{k_{z}}\left(\mathbf{r}, \mathbf{r}^{\prime}, \tau-\tau^{\prime}\right)+\text { c.c. }
$$

where $G_{k_{z}}\left(\mathbf{r}, \mathbf{r}^{\prime}, \tau-\tau^{\prime}\right)$ is the Green's function of the linearized TDGL equation (2.7) in the presence of the scalar potential describing the electric field. The correction to the Green's function to linear order in the time dependent homogeneous electric field $\mathscr{E}(\tau)$ is as follows:

$$
G_{k_{z}}\left(\mathbf{r}, \mathbf{r}^{\prime}, \tau-\tau^{\prime}\right)=G_{k_{z}}^{0}\left(\mathbf{r}, \mathbf{r}^{\prime}, \tau-\tau^{\prime}\right)-\mathrm{i} \int \mathrm{d} \mathbf{r}_{1} \int \mathrm{d} \tau_{1} G_{k_{z}}^{0}\left(\mathbf{r}, \mathbf{r}_{1}, \tau-\tau_{1}\right) \mathscr{E}\left(\tau_{1}\right) y_{1} G_{k_{z}}^{0}\left(\mathbf{r}_{1}, \mathbf{r}^{\prime}, \tau_{1}-\tau^{\prime}\right) .
$$

Substituting the full Green function (4.2) into expression (4.1), and performing the integrals, one obtains:

$$
\begin{aligned}
j_{y}^{\mathrm{s}}(\tau)= & \frac{b}{4 \pi s} \frac{\eta t}{b^{2}+\omega^{2}} \mathscr{E} \int_{0}^{\infty} \mathrm{d} \bar{\tau} \exp \left[-\left(2 \varepsilon-b+\frac{2}{d^{2}}\right) \bar{\tau}\right] I_{0}\left(\frac{2 \bar{\tau}}{d^{2}}\right) \operatorname{csch}(b \bar{\tau}) \\
& \times\{b \cos (\tau \omega) \cos (b \bar{\tau})-b \cos [(\tau-\bar{\tau}) \omega] \operatorname{csch}(b \bar{\tau})+\omega \sin (\tau \omega)\}
\end{aligned}
$$

The real and imaginary parts of the complex conductivity after the Fourier transform

$$
\sigma_{\mathrm{S}}(\omega)=\frac{j_{\mathrm{S}}(\omega)}{\mathscr{E}(\omega)}=\sigma_{1}(\omega)+\mathrm{i} \sigma_{2}(\omega)
$$

therefore, are as follows:

$$
\begin{gathered}
\sigma_{1}(\omega)=\frac{b^{2}}{8 \pi s} \frac{\eta t}{b^{2}+\omega^{2}} \int_{0}^{\infty} \mathrm{d} \bar{\tau} \exp \left[-\left(2 \varepsilon-b+\frac{2}{d^{2}}\right) \bar{\tau}\right] I_{0}\left(\frac{2 \bar{\tau}}{d^{2}}\right) \operatorname{csch}^{2}(b \bar{\tau})[\cosh (b \bar{\tau})-\cos (\omega \bar{\tau})], \\
\sigma_{2}(\omega)=\frac{b}{8 \pi s} \frac{\eta t}{b^{2}+\omega^{2}} \int_{0}^{\infty} \mathrm{d} \bar{\tau} \exp \left[-\left(2 \varepsilon-b+\frac{2}{d^{2}}\right) \bar{\tau}\right] I_{0}\left(\frac{2 \bar{\tau}}{d^{2}}\right) \operatorname{csch}^{2}(b \bar{\tau})[\omega \sinh (b \bar{\tau})-b \sin (\omega \bar{\tau})] .
\end{gathered}
$$

This is the main result of the paper. The complex resistivity of the layered material is (after noting that superconducting layers constitute a fraction $s / d$ of the material)

$$
\sigma(\omega)=\frac{s}{d} \sigma_{\mathrm{s}}(\omega)+\sigma_{n}(\omega)
$$

Neglecting the normal part, one obtains resistivity:

$$
\rho_{\mathrm{s}}(\omega)=\frac{d}{s \sigma_{\mathrm{s}}(\omega)}=\rho_{1}-\mathrm{i} \rho_{2}
$$

where

$$
\rho_{1}=\frac{d}{s} \frac{\sigma_{1}}{\sigma_{1}^{2}+\sigma_{2}^{2}}, \quad \rho_{2}=\frac{d}{s} \frac{\sigma_{2}}{\sigma_{1}^{2}+\sigma_{2}^{2}} .
$$

The difference between the conductivities in a zero field and in the field of $B$ is defined as follows:

$$
\delta \sigma=\sigma(0)-\sigma(B)
$$




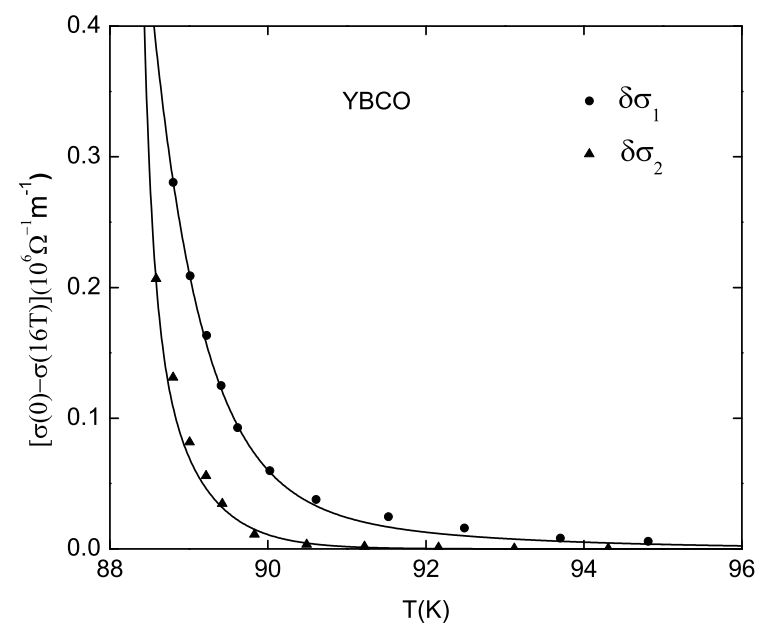

Figure 1. Points are the difference between the conductivities in zero field and in the field of $16 \mathrm{~T}\left(\delta \sigma_{1}\right.$ black circles, $\delta \sigma_{2}$ black triangles) of slightly underdoped YBCO. The solid lines are the theoretical values of resistivity for different temperatures at frequency $\omega / 2 \pi=15.15 \mathrm{GHz}$ calculated from equation (4.10) with fitting parameters (see text).

\section{Comparison with experiment}

The experimental results by M.S. Grbić et al. [19], obtained from the the microwave absorption measurements at $\omega / 2 \pi=15.15 \mathrm{GHz}$ on slightly underdoped $\mathrm{YBa}_{2} \mathrm{Cu}_{3} \mathrm{O}_{7-\delta}$ (YBCO) with $T_{\mathrm{c}}=87 \mathrm{~K}$. The distance between the bilayers using the calculation is $d^{\prime}=11.68 \AA$ in [24]. In order to compare the fluctuation conductivity with experimental data in HTSC, one cannot use the expression of relaxation time $\gamma^{\prime}$ in Bardeen-Cooper-Schrieffer theory which may be suitable for a low- $T_{\mathrm{c}}$ superconductor. Instead of this, we use the factor $k$ as a fitting parameter. The comparison is presented in figure 1 The ac conductivity curves were fitted to equation (4.10) with the normal-state conductivity measured in [17] to be $\sigma_{n}=3.3 \cdot 10^{6}(\Omega \mathrm{m})^{-1}$. The parameters we obtain from the fit are: $H_{\mathrm{c} 2}(0)=T_{\mathrm{c}} d H_{\mathrm{c} 2}(T) /\left.d T\right|_{T_{\mathrm{c}}}=178 \mathrm{~T}$ (corresponding to $\xi=13.6 \AA$ ), the GL parameter $\kappa=49.7$, the order parameter effective thickness $s^{\prime}=5.51 \AA$, and the factor $k=\sigma_{n} / \sigma_{\mathrm{GL}}=0.86$, where we take $\gamma=10$ for YBCO in [25]. Using those parameters, we obtain $\mathrm{Gi}=2.78 \cdot 10^{-3}$ (corresponding to $\eta=0.176$ ). The order parameter effective thickness $s^{\prime}$ can be taken to be equal to the layer distance (see in [26]) of the superconducting $\mathrm{CuO}_{2}$ plane plus the coherence length $2 \xi_{\mathrm{c}}=2 \frac{\xi}{\gamma}$ due to the proximity effect: $3.18 \AA+2 \frac{13.6}{10} \AA=4.54 \AA$, roughly in agreement in magnitude with the fitting value of $s^{\prime}$.

\section{Discussion and conclusion}

The complex conductivity was calculated in a layered type-II superconductor under magnetic field in the presence of strong thermal fluctuations on the mesoscopic scale in linear response. While in the normal state, the dissipation involves unpaired electrons, in the mixed phase it takes a form of the flux flow. Time dependent Ginzburg-Landau equations with thermal noise describing the thermal fluctuations are used to describe the vortex-liquid regime. The nonlinear term in dynamics is treated using the renormalized Gaussian approximation. Explicit expressions for the complex conductivity $\sigma_{\mathrm{s}}$ and resistivity $\rho_{\mathrm{s}}$ including all Landau levels were obtained, therefore the approach is valid for arbitrary values if the magnetic field is not too close to $H_{\mathrm{cl}}(T)$.

The results were compared to the experimental data on HTSC. The results are in good qualitative and even quantitative agreement with experimental data on YBCO. The thermal fluctuation was included in the present approach, so the results should be applicable for above and below $T_{\mathrm{c}}$. 


\section{Acknowledgements}

We are grateful to Baruch Rosenstein, Dingping Li for discussions. This work was supported by the National Foundation for Science and Technology Development (NAFOSTED) of Vietnam under Grant No. 103.02-2011.15.

\section{References}

1. Xu Z.A., Ong N.P., Wang Y., Kakeshita T., Uschida S., Nature, 2000, 406, 486; doi $10.1038 / 35020016$

2. Dorin V.V., Klemm R.A., Varlamov A.A., Buzdin A.I., Livanov D.V., Phys. Rev. B, 1993, 48, 12951; doi 10.1103/PhysRevB.48.12951

3. Ussishkin I., Sondhi S.L., Huse D.A., Phys. Rev. Lett., 2002, 89, 287001; doi 10.1103/PhysRevLett.89.287001

4. Li L., Wang Y., Komiya S., Ono S., Ando Y., Gu G.D., Ong N.P., Phys. Rev. B, 2010, 81, 054510; doi 10.1103/PhysRevB.81.054510

5. Larkin A., Varlamov A., Theory of Fluctuations in Superconductors, Clarendon Press, Oxford, 2005.

6. Rosenstein B., Li D., Rev. Mod. Phys., 2010, 82, 109; doi 10.1103/RevModPhys.82.109

7. Schmidt H., Z. Phys., 1968, 216, 336; doi 10.1007/BF01391528

8. Schmidt H., Z. Phys., 1970, 232, 443; doi 10.1007/BF01395675

9. Dorsey A.T., Phys. Rev. B, 1991, 43, 7575; doi 10.1103/PhysRevB.43.7575

10. Wickham R.A., Dorsey A.T., Phys. Rev. B, 2000, 61, 6945; doi 10.1103/PhysRevB.61.6945

11. Fisher D.S., Fisher M.P.A., Huse D.A., Phys. Rev. B, 1991, 43, 130; doi 10.1103/PhysRevB.43.130

12. Ong N.P., Wu H., Phys. Rev. B, 1997, 56, 458; doi 10.1103/PhysRevB.56.458

13. Maniv T., Rosenstein B., Shapiro I., Shapiro B.Ya., Phys. Rev. B, 2009, 80, 134512; doi 10.1103/PhysRevB.80.134512

14. Lin P.-J., Lipavsky P., Phys. Rev. B, 2009, 80, 212506; doi 10.1103/PhysRevB.80.212506

15. Tinh B.D., Thu L.M., Mod. Phys. Lett. B, 2012, 26, 1250143; doi $10.1142 / S 0217984912501436$

16. Tinh B.D., Physica C, 2013, 485, 10; doi 10.1016/j.physc.2012.10.005

17. Tsuchiya Y., Iwaya K., Kinoshita K., Hanaguri T., Kitano H., Maeda A., Shibata K., Nishizaki T., Kobayashi N., Phys. Rev. B, 2001, 63, 184517; doi 10.1103/PhysRevB.63.184517.

18. Hanaguri T., Tsuboi T., Tsuchiya Y., Sasaki K.I., Maeda A., Phys. Rev. Lett., 1999, 82, 1273; doi 10.1103/PhysRevLett.82.1273

19. Grbić M.S., Požek M., Paar D., Hinkov V., Raichle M., Haug D., Keimer B., Barišić N., Dulčić A., Phys. Rev. B, 2011, 83, 144508; doi 10.1103/PhysRevB.83.144508

20. Ketterson J.B., Song S.N., Superconductivity, Cambridge University Press, Cambridge, 1999.

21. Rosenstein B., Zhuravlev V., Phys. Rev. B, 2007, 76, 014507; doi 10.1103/PhysRevB.76.014507

22. Kopnin N., Vortices in Type-II Superconductors: Structure and Dynamics, Oxford University Press, Oxford, 2001.

23. Tinh B.D., Li D., Rosenstein B., Phys. Rev. B, 2010, 81, 224521; doi 10.1103/PhysRevB.81.224521

24. Yan Y., Blanchin M.G., Phys. Rev. B, 1991, 43, 13717; doi 10.1103/PhysRevB.43.13717

25. Li D., Rosenstein B., Phys. Rev. B, 2002, 65, 220504(R); doi 10.1103/PhysRevB.65.220504.

26. Poole C.P.(Jr.), Farach H.A., Creswick R.J., Prozorov R., Superconductivity, Academic Press, Amsterdam, 2007. 


\title{
Комплексна провідність у сильно флуктуюючих шаруватих надпровідниках
}

\author{
Д.Тінь, Л.М. Тхю, Л.Б. Хоа \\ Фізичний факультет, національний університет освіти м. Ханой, 136 Сюентхуі, Канзяі, Ханой, В'єтнам \\ Часозалежним методом Гінзбурга-Ландау розраховано комплексну флуктуаційну провідність у шарува- \\ тому надпровіднику II-го типу під дією магнітного поля. Шарувата структура надпровідника враховується \\ за допомогою моделі Лоуренса-Доняха, тоді як член, що описує нелінійну взаємодію в динаміці, розгля- \\ дається у самоузгодженому гаусовому наближенні. У високотемпературних матеріалах значна частина \\ діаграми $H-T$ належить до вихорової рідинної фази. Вирази для сумарного внеску від усіх рівнів Ландау \\ записані в явній формі. Вони застосовні по суті до всієї цієї фази і порівнюються з експериментальними \\ даними для високотемпературного надпровідника $\mathrm{YBa}_{2} \mathrm{Cu}_{3} \mathrm{O}_{7-\delta}$. Вище кросовера до "нормальної фази" \\ наші результати узгоджуються з раніше отриманими.
}

Ключові слова: часозалежний метод Гінзбурга-Ландау, комплексна провідність, надпровідник II-го типу 